\title{
Reproducción de Saccodon dariensis (Teleostei: Parodontidae) en afluentes del río Guatapé, cuenca del río Magdalena, Colombia
}

\author{
Néstor Javier Mancera-Rodríguez ${ }^{1}$, Jeiner Castellanos-Barliza ${ }^{1,2} \&$ Diego Urrego-Ballestas ${ }^{1}$ \\ 1. Universidad Nacional de Colombia, Sede Medellín. Departamento de Ciencias Forestales, Grupo Ecología y \\ Conservación de Fauna Silvestre, Calle 59A No. 63-20, Bloque 20, oficina 211, Medellín, Colombia; \\ njmancer@unal.edu.co, urrego-ballestas@hotmail.com \\ 2. Universidad del Magdalena; Grupo en Ecología Neotropical; Carrera 32 \#22-08, Santa Marta, Magdalena, Colombia; \\ jeinercast@gmail.com
}

Recibido 30-VII-2015. Corregido 13-XII-2015. Aceptado 13-I-2016.

\begin{abstract}
Reproduction of Saccodon dariensis (Teleostei: Parodontidae) in Guatapé River tributaries, Magdalena River basin, Colombia. The Andean mountain region of Colombia has a high diversity of fish, with high number of endemic species. To promote their protection and conservation, the knowledge of their general and reproductive biology is necessary. With this aim, the reproductive biology of Saccodon dariensis, in the Peñoles and El Cardal creeks, Guatape River mid-basin, Magdalena River Basin, was studied, to determine reproductive differences between individuals with different oral polymorphism (morpho I vs. morpho IV, according to Roberts, 1974), and to define a possible spatial or temporal reproductive isolation. Ten field samplings were carried out between October 2007 and February 2012, in periods of rain (October and November 2007, May 2008 and November 2011), dry (January and March 2010, February 2012), transition from rain to dry (June 2011) and transition from dry to rain (March 2008 and September 2011). Samples were caught using electrofishing equipment and cast nets (10 mm between knots). A total of 468 specimens were analyzed, 268 of which were females and 200 males. The average catch size for the total number of individuals studied was 109.6 mm SL (65.5-174.0 mm), with 108.0 mm SL for females (67.7-174.0) and 111.9 mm SL (65.5-149.4) for males. Females predominated in the catches, and the sex ratio of 1.0:1.34 significantly deviated from the theoretical distribution 1:1. Similarly, morpho IV predominated in the catches, and morphs ratio was 1.00:1.48. Based on the monthly evolution of the gonadosomatic index (GSI) and the proportion of mature specimens, the spawning season occurs during periods of transition from dry to rainy season when water level begins to raise. The lowest values of the condition factor that match the maximum values of GSI, indicates that this species accumulated body energy reserves that are used during the gonadal maturation and spawning. The mean size at sexual maturity $\left(\mathrm{L}_{50}\right)$ was $88.8 \mathrm{~mm} \mathrm{SL}$ in females and $109.3 \mathrm{~mm}$ SL in males. The fecundity of $S$. dariensis fluctuated between 1137 and 39303 (mean = 8309, SD =9 021) and the relative fecundity between 144 and 1131 oocytes $/ g$ of total weight $($ mean $=439 \pm 212)$. The diameter of the oocytes was $0.54 \mathrm{~mm}(\mathrm{SD}=0.07)$. Different development in oocytes was not observed, suggesting massive spawning. The coexistence of the two morphs, external fertilization and simultaneous occurrence of reproductive peaks found in this study did not provide support for a possible spatial or temporal reproductive isolation of morphotypes. Rev. Biol. Trop. 64 (2): 635-653. Epub 2016 June 01.
\end{abstract}

Key words: conservation, reproductive aspects, tropical freshwater fish, means size at sexual maturity.

El éxito reproductivo de los peces depende de dónde y cuándo se reproducen y de los recursos asignados para la reproducción (Wootton, 1984), de manera que ésta debe suceder en el período del año en que se maximiza la producción de descendencia (Rondineli \& Braga,
2010). Conocer la biología reproductiva de los peces, que incluye el período de desove, la fecundidad y la talla o edad en la que se producen estos eventos en una especie, es importante para establecer estrategias para la conservación de sus poblaciones, ya que de la reproducción 
depende el reclutamiento y el mantenimiento de poblaciones viables (Rondineli \& Braga, 2010). Así mismo, el conocimiento de las tácticas reproductivas es fundamental para comprender las estrategias del ciclo de vida de las especies, que son cruciales para direccionar medidas de gestión y conservación relacionadas con acciones humanas como la pesca, la contaminación, y la eliminación de las zonas de reproducción mediante la construcción de represas y la destrucción de la vegetación marginal (Vazzoler \& Menezes, 1992).

La familia Parodontidae está compuesta por los géneros Parodon Valenciennes 1849 y Saccodon Kner 1863 (Londoño-Burbano \& Román-Valencia, 2010), con amplia distribución geográfica en el centro y sur de América, desde el Canal de Panamá hasta la cuenca del Río de la Plata en Argentina, excepto en las cuencas del sur del Estado de Bahía en Brasil en la Costa Atlántica, la Patagonia, y el canal del río Amazonas (Pavanelli, 2003).

Saccodon dariensis (Meek \& Hildebrand, 1913), se distribuye en Centro y Suramérica, en aguas continentales al oriente del canal de Panamá y en Colombia en las cuencas de los ríos Atrato y Magdalena (Pavanelli, 2003; Maldonado-Ocampo, Vari, \& Usma-Oviedo, 2008), en particular en los departamentos de Cauca, Valle del Cauca, Quindío, Santander (UsmaOviedo et al., 2013), y Antioquia (JaramilloVilla, Maldonado-Ocampo, \& Bogotá-Gregory, 2008; Restrepo-Gómez \& Mancera-Rodríguez, 2014). Saccodon dariensis presenta dimorfismo sexual (Londoño-Burbano \& RománValencia, 2010) y los machos adultos presentan tubérculos nupciales durante la época reproductiva (Wiley \& Collette, 1970). Se encuentra asociada a sitios de corrientes rápidas, bajas pendientes y sustratos rocosos cubiertos de perifiton, y está incluida en el Libro Rojo de peces dulceacuícolas de Colombia en la categoría de Preocupación Menor (Usma-Oviedo, Ortega-Lara, \& Mancera-Rodríguez, 2012).

Roberts (1974) ha señalado cinco polimorfismos bucales y dentales en ejemplares de
Saccodon dariensis recolectados en afluentes de los ríos Sinú, Magdalena, alto Cauca y Atrato, con dientes altamente especializados para raspar algas adheridas al sustrato rocoso en los ecosistemas acuáticos. Para la cuenca del río Guatapé se ha definido la coexistencia de los morfotipos I y IV definidos por Roberts (1974), relacionados con un polimorfismo trófico que le confiere diferentes hábitos tróficos y acceso diferencial a los ítems que constituyen su dieta (Restrepo-Gómez \& Mancera-Rodríguez, 2014).

Los aspectos reproductivos de especies de la familia Parodontidae en zonas andinas han sido poco estudiados. Usma-Oviedo et al. (2013) presentaron información sobre la migración y época reproductiva de Saccodon dariensis para la cuenca alta del río Cauca, y Pareja y Jiménez-Segura (2012) describieron algunos aspectos de la biología reproductiva de Parodon magdalenensis en el río La Miel (Caldas, Colombia). Para otras zonas de Suramérica se han realizado estudios que presentan datos reproductivos en Apareiodon affinis (Godoy, 1975; Oldani, 1977; Nomura, Ferreira, \& Hayashi, 1978; Barbieri et al., 1985; Bialetzki, Sanches, Baumgartner, \& Nakatani, 1998; Fonseca-Ratton, Bazzoli, \& Bastos-Santos, 2003), A. ibitiensis (Barbieri, Verani, \& Barbieri, 1983; Barbieri et al., 1985; Rondineli \& Braga, 2010), A. piracicabae (Sazima, 1980); y Parodon nasus (Nomura, 1979; Barbieri et al., 1985; Azevedo, Barbieri, \& Barbieri, 1988a, b; Barbieri \& Barbieri, 1989; Gomiero \& Braga, 2007; Rondineli \& Braga, 2010).

El objetivo de este trabajo fue determinar las principales características reproductivas de Saccodon dariensis (nombre común local: mazorco), en afluentes de la parte media del río Guatapé, cuenca del río Magdalena, y determinar si existen diferencias reproductivas entre individuos con diferente polimorfismo bucal (morfo I - boca recta vs morfo IV - boca en media luna), que permita definir un posible aislamiento reproductivo espacial o temporal de los individuos con diferente morfotipo. 


\section{MATERIALES Y MÉTODOS}

Localización y descripción del sitio de estudio: El trabajo se realizó en tres estaciones de la quebrada Peñoles (E1: 6¹5'49.6" N - 7505'06.4" W, 1265 m.s.n.m.; E2: 6 16 '04.4" N - 7505'12.7" W, 1246 m.s.n.m.; E3: 6०16'27.2" N - 75'05'23.7” W, 1201 m.s.n.m.) y tres estaciones de la quebrada El Cardal (E1: 6 ${ }^{\circ} 16^{\prime} 47.5^{\prime \prime} \mathrm{N}-74^{\circ} 566^{\prime 2} 27.4^{\prime}$ W, 944 m.s.n.m.; E2: 6016'37.5" N - 74'55'47.9" W, 940 m.s.n.m.; E3: 6º'16'46.6” N - 7455'24.6" W, 898 m.s.n.m.), afluentes del río Guatapé en los municipios de San Rafael y San Carlos, Antioquia, Colombia. La zona presenta características de bosque húmedo tropical montano bajo (Holdridge, 1967), zonobioma húmedo tropical del valle del río Magdalena, con una temperatura promedio entre 23 y $28^{\circ} \mathrm{C}$ (Rodríguez, Armenteras, Morales, \& Romero, 2004), y un patrón de precipitación bimodal con picos en mayo y octubre, con un promedio anual de $5720 \mathrm{~mm}$ de lluvia para la estación La Araña (2308067 - Red Hidrometeorológica de Empresas Públicas de Medellín EE.PP.M) ubicada cerca a la Quebrada Peñoles y de 4 368mm para la estación Embalse de Playas (2308799 Red Hidrometeorológica de EE.PP.M) ubicada cerca a la quebrada El Cardal. El territorio es montañoso, con topografía ondulada, y las quebradas drenan hacia la cuenca media del río Magdalena, caracterizándose por ser torrenciales, de aguas muy claras y sin planicie aluvial.

Caracterización del hábitat: Para la caracterización del hábitat se midieron las siguientes variables fisicoquímicas in situ: oxígeno disuelto $(\mathrm{mg} / \mathrm{L})$, saturación de oxígeno $(\%)$, temperatura superficial del agua $\left({ }^{\circ} \mathrm{C}\right)$ con medidor Hanna (HI9828/4-01) y pH, conductividad $(\mu \mathrm{S} / \mathrm{cm})$ y sólidos totales disueltos (STD) con medidor Hanna (9811-5). Ancho y profundidad fueron medidos con flexómetro, y una vara graduada en $\mathrm{cm}$. El tipo de sustrato se clasificó de acuerdo con la escala propuesta por Wentworth (1922).

Obtención de las muestras: Se realizaron diez muestreos entre octubre 2007 y febrero
2012, que incluyeron períodos de lluvia (octubre y noviembre 2007, mayo 2008 y noviembre 2011), períodos secos (enero y marzo 2010, febrero 2012), transición de lluvia a seco (junio 2011) y transición de seco a lluvia (marzo 2008 y septiembre 2011). En cada punto se realizó pesca con un equipo de electropesca SAMUS $725 \mathrm{MP}$ el cual se usó con descargas en un tramo lineal de $100 \mathrm{~m}$ por estación, haciendo barridos en contra de la corriente, y de ribera a ribera, que incluyera todos los biotopos posibles para evaluar la presencia de la especie, y 30 lances de atarraya de $1 \mathrm{~cm}$ ojo de malla por estación.

Los 468 ejemplares capturados se fijaron en formol al $10 \%$ y se etiquetaron con fecha, lugar de captura y arte de pesca. La identificación de estadios sexuales se realizó en el laboratorio de Ecología y Conservación de Fauna Silvestre de la Universidad Nacional de Colombia, Sede Medellín. En el laboratorio, los ejemplares fueron lavados y preservados en alcohol al $70 \%$. Posteriormente, se midió su longitud estándar $(\mathrm{mm})$ con un calibrador digital hasta centésimas de milímetro, y su peso total (g) y eviscerado (g) se tomaron con una balanza analítica (Ohaus Scout-Pro 202, 0.01 g). Los ejemplares se evisceraron uroventralmente para la extracción de las gónadas, las cuales fueron pesadas (g), y fijadas en formol al $5 \%$ y depositadas en frascos rotulados con la información del ejemplar. Ejemplares de $S$. dariensis se preservaron y están depositados en los Laboratorios de Ictiología de la Universidad del Quindío, Armenia, Colombia (IUQ) y en la colección de peces de la Universidad Católica de Oriente (Colombia), inscrita en el Instituto de Investigación de Recursos Biológicos Alexander von Humboldt Colombia, con código CP-UCO.

Se estimó la relación longitud estándar (LE)-peso total (PT) que se ajustó a un modelo tipo potencial, de acuerdo a la ecuación: $\log \mathrm{PT}=\log a+b \operatorname{logLE}$, donde $a$ es una constante de la regresión equivalente al factor de condición (Kn), y $b$ el coeficiente de crecimiento alométrico. Parámetros $(a)$ y $(b)$, y el nivel de significancia estadística de $\mathrm{R}^{2}$ fueron determinados por un análisis de regresión 
lineal ajustado mediante el método de mínimos cuadrados (Zar, 1999). Los límites de confianza del $95 \%$ para b (LC $95 \%$ ) se calcularon utilizando la siguiente ecuación: $\mathrm{LC}=b \pm(1.96$ x DE), donde $\mathrm{DE}$ es la desviación estándar de $b$. Para comprobar si el valor de $\mathrm{b}$ fue significativamente diferente de 3 , se realizó una prueba $\mathrm{t}$ de Student de acuerdo con Sokal y Rohlf (1987). Para contrastar diferencias de longitud estándar y peso total entre sexos y polimorfismos bucales, se utilizó la prueba no paramétrica de Kruskall-Wallis y las comparaciones pareadas con la prueba Mann-Whitney. Estas pruebas se utilizaron en lugar de pruebas paramétricas, debido a que los datos de tamaño no cumplieron con los supuestos de normalidad y homocedasticidad.

La proporción de sexos (machos: hembras) se estableció para cada período de muestreo y para cada clase de longitud, y se utilizó una prueba de Chi-cuadrado $\left(\chi^{2}\right)$ para determinar diferencias significativas. La distribución de tallas se estimó en intervalos de $10 \mathrm{~mm}$ de longitud estándar. Se determinó el sexo y el estadio de desarrollo gonadal de cada individuo mediante análisis macroscópico de las gónadas, siguiendo la escala de maduración propuesta por Holden y Raitt (1975), (I: Inmaduro, II: Reposo, III: En maduración, IV: Maduro y V: Desovado (hembras) o espermiado (machos). El tiempo de la actividad reproductiva se evaluó mediante los cambios en la condición gonadal a través del tiempo, para lo que se calculó el índice gonadosomático (IGS) (Vazzoler, 1996): $\mathrm{IGS}=(\mathrm{Pg} / \mathrm{Pt}) \times 100$ donde, $\mathrm{Pg}$ es el peso de la gónada y Pt es el peso total del pez en gramos. Este índice fue calculado para cada pez, y todos los valores se promediaron por período de muestreo.

Para determinar el grado de bienestar del pez, se calculó el factor de condición $(\mathrm{K})$, según Anderson y Gutreuter (1983): K= Pe/ $\mathrm{a}(\mathrm{LE})^{\mathrm{b}}$ donde, $\mathrm{Pe}$ es el peso eviscerado del ejemplar y $\mathrm{a}(\mathrm{LE})^{\mathrm{b}}$ es el peso teórico eviscerado para peces de la misma longitud, obtenido a partir de la relación longitud estándar-peso. El índice hepatosomático (IHS) se calculó para cada ejemplar mediante la fórmula: $\mathrm{IHS}=(\mathrm{Ph} /$
PT-Ph) x 100, donde Ph es el peso del hígado y PT es el peso total del pez; todos los pesos se midieron en gramos.

Para estimar la talla media de madurez sexual $\left(\mathrm{LE}_{50}\right)$, se calculó la proporción (Pi) de individuos maduros por sexo y por clase de longitud: $\mathrm{Pi}=(\mathrm{Mi} / \mathrm{Iei}) \times 100$, donde $\mathrm{Mi}=$ número de individuos maduros en la longitud i; Iei = número de individuos examinados en la clase de talla i. Los datos obtenidos se ajustaron a una función logística utilizando el programa estadístico PAST (Hammer y Harper, Oslo, Noruega) (Hammer, Harper, \& Ryan, 2001). La ecuación utilizada fue la siguiente: $\mathrm{P}=1 /$ $\left(1+\mathrm{e}^{-\mathrm{r}(\mathrm{L}-\mathrm{L} 50)}\right)$; donde $\mathrm{L}$ es la longitud estándar correspondiente a la proporción (P), $\mathrm{r}$ es una constante que indica la pendiente inicial de la curva y $\mathrm{L}_{50}$ es la longitud en la cual el $50 \%$ de los individuos están maduros.

La fecundidad se estimó como el recuento total de ovocitos maduros en los ovarios de una muestra de 63 hembras maduras, en un rango de 67.7 hasta $158.0 \mathrm{~mm}$ de LE y con un IGS que varió entre 5.05 y 25.68 durante la temporada de desove. Las gónadas maduras se conservaron en fluido Gilson y se utilizó el método gravimétrico, en el que la fecundidad se determina como el producto del peso de las gónadas y la densidad de los ovocitos (número de ovocitos por gramo de tejido de ovario), y se determinó contando el número de ovocitos en una muestra pesada de tejido ovárico. Los ovarios se pesaron y cinco submuestras fueron extraídas de diferentes partes del ovario. Cada submuestra se pesó a $0.001 \mathrm{~g}$, se dispersó con un pincel fino y se contaron los ovocitos. El diámetro de 100 ovocitos tomados al azar, se midió utilizando un microscopio estereoscópico con una reglilla.

El número total de ovocitos maduros $(\mathrm{F})$ en los ovarios (fecundidad absoluta) fue calculado utilizando la siguiente fórmula: $\mathrm{F}=$

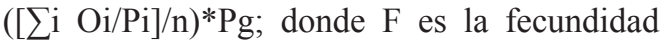
absoluta, Oi es el número de ovocitos, Pi es el peso submuestra, Pg es el peso de la gónada y $\mathrm{n}$ el número de submuestras. Se estimó la relación entre la fecundidad absoluta (el número de ovocitos maduros por peso) y la longitud 
estándar, el peso total, el peso eviscerado y el peso de la gónada.

\section{RESULTADOS}

Características del hábitat: La quebrada Peñoles presenta sustrato pedregoso, con pendiente inclinada y rocas de gran tamaño dentro y en su margen, con aguas frías que durante la época seca son cristalinas y en época de lluvias turbias y corrientosas. El sitio E1 está ubicado en la parte alta de la quebrada, la ribera derecha presenta predominio de bosque nativo $\mathrm{y}$ la izquierda tiene cobertura boscosa y algunos potreros. El sitio E2 se encuentra en la parte media y en sus riberas se encuentran pastos y vegetación nativa, y el punto $\mathbf{E 3}$ se ubica cerca de la desembocadura en el río Guatapé, y en sus riberas predomina el bosque nativo y algunos potreros para ganadería. Cincuenta metros antes de su desembocadura se observa una vía de concreto que cruza la quebrada para el acceso de vehículos a una finca. No se observaron medidas artificiales de protección de riberas, ni presencia de aceites $\mathrm{u}$ olores particulares en el sedimento o el agua. La temperatura superficial del agua presentó un promedio de $19.3{ }^{\circ} \mathrm{C}$, el oxígeno disuelto de $10.5 \mathrm{mg} / \mathrm{L}$, el porcentaje de saturación de oxígeno de 123.7 $\%$, la conductividad de $17.9 \mu \mathrm{S} / \mathrm{cm}$, los sólidos totales disueltos de $17.3 \mathrm{y}$ el $\mathrm{pH}$ de 7.8. En general, la quebrada presentó una profundidad media entre $0.40-0.60 \mathrm{~m}$, aunque con algunas pozas de mayor profundidad. El ancho promedio fue de $14.9 \mathrm{~m}$ con máximo de $16.6 \mathrm{~m}$ en época de lluvias y mínimo de $13.1 \mathrm{~m}$ en época seca (Cuadro 1).

La quebrada El Cardal presenta sustrato arenoso-pedregoso, con rocas de gran tamaño dentro de la quebrada. Durante la época seca sus aguas son claras y en época de lluvias turbias con carga de sedimentos y corrientosas. El sitio E1 presenta rocas de gran tamaño con la mayor parte de su área expuesta y en ambas riberas presencia de bosque nativo. Se observan actividades de minería artesanal de oro en sus márgenes. El sitio E2 se encuentra en la parte media de la quebrada y en sus riberas predomina la vegetación nativa, y el punto E3 se ubica $200 \mathrm{~m}$ antes de la desembocadura en el río Guatapé, en ambas riberas el uso del suelo predominante es de pastos para ganadería. No se observan medidas artificiales de protección de riberas, ni presencia de aceites en el sedimento o el agua. Los sedimentos presentaron olor a materia orgánica en descomposición y presencia de espumas, el agua se observó turbia y en el sitio E3 se presentan olores fuertes debido a vertimientos que se realizan desde un campamento habitacional de una empresa generadora de energía, ubicado en el margen derecha de la quebrada, igualmente presencia

CUADRO 1

Valores promedio de los parámetros físicoquímicos del agua para las diferentes estaciones de muestreo en las quebradas Peñoles y El Cardal, en la parte media del río Guatapé, Oriente antioqueño, Colombia

TABLE 1

Average values of Physical-chemical parameters of water for different sampling stations in the Peñoles and El Cardal streams in the Guatapé River mid-basin, Eastern Antioquia, Colombia

\begin{tabular}{lcccccccc}
\multicolumn{1}{c}{ Variable } & \multicolumn{3}{c}{ Quebrada Peñoles } & \multicolumn{5}{c}{ Quebrada E1 Cardal } \\
\multicolumn{1}{c}{ Estación } & E1 & E2 & E3 & Media & E1 & E2 & E3 & Media \\
Temperatura agua $\left({ }^{\circ} \mathrm{C}\right)$ & 18.9 & 19.5 & 19.6 & 19.3 & 20.4 & 21.8 & 21.4 & 21.2 \\
Oxígeno disuelto $(\mathrm{mg} / \mathrm{L})$ & 10.4 & 11.0 & 10.2 & 10.5 & 8.1 & 10.7 & 8.1 & 9.0 \\
Saturación de oxígeno $(\%)$ & 120.2 & 131.5 & 119.5 & 123.7 & 99.3 & 126.1 & 93.4 & 106.3 \\
Conductividad $(\mu \mathrm{S} / \mathrm{cm})$ & 18.8 & 17.5 & 17.3 & 17.9 & 28.6 & 34.2 & 40.3 & 34.4 \\
Sólidos totales disueltos & 21.3 & 14.3 & 16.3 & 17.3 & 30.0 & 34.3 & 42.3 & 35.5 \\
pH & 7.9 & 8.0 & 7.5 & 7.8 & 7.7 & 7.8 & 7.7 & 7.7 \\
Ancho $(\mathrm{m})$ & 14.6 & 13.7 & 16.5 & 14.9 & 11.7 & 11.0 & 11.6 & 11.4 \\
\hline
\end{tabular}


de residuos sólidos. La temperatura superficial del agua presentó un promedio de $21.2^{\circ} \mathrm{C}$, el oxígeno disuelto de $9.0 \mathrm{mg} / \mathrm{L}$, el porcentaje de saturación de oxígeno de $106 \%$, la conductividad de $34.4 \mu \mathrm{S} / \mathrm{cm}$, los sólidos totales disueltos de 35.5 y el pH de 7.7 (Cuadro 1). La quebrada presentó una profundidad media entre 0.30 $0.60 \mathrm{~m}$, aunque con algunas pozas de mayor profundidad. El ancho promedio fue de $11.4 \mathrm{~m}$ con máximo de $12.9 \mathrm{~m}$ en época de lluvias y mínimo de $9.8 \mathrm{~m}$ en época seca.

Distribución de las capturas: En total se capturaron 468 ejemplares de Saccodon dariensis; 250 ejemplares $(53.4 \%$ del total $)$ en la quebrada Peñoles y 218 ejemplares en la quebrada El Cardal (46.6 \%). Con relación a los morfotipos se capturaron 189 ejemplares con morfotipo I y 279 con morfotipo IV. Por sexo se capturaron 268 hembras y 200 machos.

Características de los ejemplares: Los individuos de $S$. dariensis estudiados fueron peces en un rango entre 65.5 y $174 \mathrm{~mm}$ de LE y peso total (PT) entre 3.7 y $114.9 \mathrm{~g}$, presentan las hembras un rango entre 67.7 y $174 \mathrm{~mm}$ de LE y PT entre 4.8 y $114.9 \mathrm{~g}$ y los machos un rango entre 65.5 y $149.4 \mathrm{~mm}$ de LE y PT entre 3.7 y $73.1 \mathrm{~g}$ (Cuadro 2). Las medianas de LE y de PT no fueron significativamente diferentes entre sexos $(\mathrm{P}=0.054$; y $\mathrm{P}=0.051$, respectivamente), mientras que entre morfotipos bucales si lo fueron ( $\mathrm{P}<0.001$; y $\mathrm{P}<0.001$, para LE y $\mathrm{PT}$ respectivamente), siendo los ejemplares con morfotipo IV más grandes y con mayor peso.

El tipo de crecimiento, determinado por la prueba t de Student, reveló que $S$. dariensis muestra un crecimiento alométrico positivo (b $>3$ ), siendo favorecido el peso total sobre la longitud estándar a lo largo del crecimiento, con constantes alométricas (b) de 3.45 para hembras y de 3.62 para machos, y por morfotipo bucal de 3.50 para individuos con morfotipo I y de 3.59 para individuos con morfotipo IV (Cuadro 2).

Proporción de sexos y morfotipos bucales: La proporción de sexos fue de 1.00:1.34 (200 machos y 268 hembras), que se desvió significativamente de la hipotética distribución de $1: 1\left(X^{2}=9.88, P=0.007\right)$. La proporción de morfotipos fue de 1.00:1.48 (189 individuos con morfotipo I y 279 con morfotipo IV), que también se desvió significativamente $\left(X^{2}=\right.$ 17.31, $\mathrm{P}<0.001)$. De acuerdo con la distribución de los sexos con relación a la longitud estándar, las hembras fueron significativamente más abundantes en tamaños menores a los $109.9 \mathrm{~mm}$ y mayores a $130 \mathrm{~mm}$ de LE, mientras que los machos fueron más frecuentes en el

CUADRO 2

Relación longitud estándar - peso total para Saccodon dariensis por sexo y morfotipo oral en la parte media del río Guatapé, Oriente antioqueño, Colombia

TABLE 2

Length standard - Weight (LS-W) relationships for Saccodon dariensis by sex and oral morphotype in the Guatapé River mid-basin, Eastern Antioquia, Colombia

\begin{tabular}{lccccccccccccc} 
& \multicolumn{4}{c}{ Longitud estándar $(\mathrm{mm})$} & \multicolumn{4}{c}{ Peso total $(\mathrm{g})$} & \multicolumn{5}{c}{ Confidence interval for b (95\%) } \\
\multicolumn{1}{c}{ Sexo } & $\mathrm{N}$ & $\mathrm{X}$ & Min. & Max. & $\mathrm{X}$ & Min. & Max. & $\mathrm{a}$ & $\mathrm{b}$ & Min. & Max. & $\mathrm{R}^{2}$ \\
Hembras & 268 & 108.0 & 67.7 & 174.0 & 26.6 & 4.8 & 114.9 & 0.000001 & 3.45 & 3.39 & 3.50 & 0.963 \\
Machos & 200 & 111.9 & 65.5 & 149.4 & 29.9 & 3.7 & 73.1 & 0.000002 & 3.62 & 3.57 & 3.67 & 0.969 \\
Total & 468 & 109.6 & 65.5 & 174.0 & 28.0 & 3.7 & 114.9 & 0.000002 & 3.52 & 3.46 & 3.57 & 0.965 \\
Morfo & & & & & & & & & & & & & \\
I & 189 & 102.2 & 67.7 & 152.6 & 22.5 & 4.7 & 64.7 & 0.000001 & 3.59 & 3.53 & 3.65 & 0.961 \\
IV & 279 & 114.7 & 65.5 & 174.0 & 31.8 & 3.7 & 114.9 & 0.000002 & 3.50 & 3.45 & 3.55 & 0.964 \\
\hline
\end{tabular}

$\mathrm{N}$ : número de individuos; X: promedio; Min: mínimo; Max: máximo; $\mathrm{R}^{2}$ : coeficiente de determinación; a y b: parámetros de relación. 
intervalo entre los 110 y los $129.9 \mathrm{~mm}$ de LE $\left(X^{2}=88.01, \mathrm{P}<0.001, \mathrm{gl}=10\right)$ (Fig. 1A). Por morfotipo bucal, la distribución con relación a la longitud estándar muestra que el morfotipo I fue significativamente más abundante en tamaños menores a los $79.9 \mathrm{~mm}$ de LE y el morfotipo IV fue más frecuente en ejemplares mayores a $80 \mathrm{~mm}$ de LE, principalmente, por encima de los $110 \mathrm{~mm}$ de LE $\left(X^{2}=68.01, \mathrm{P}<\right.$ 0.001, gl=10) (Fig. 1B).
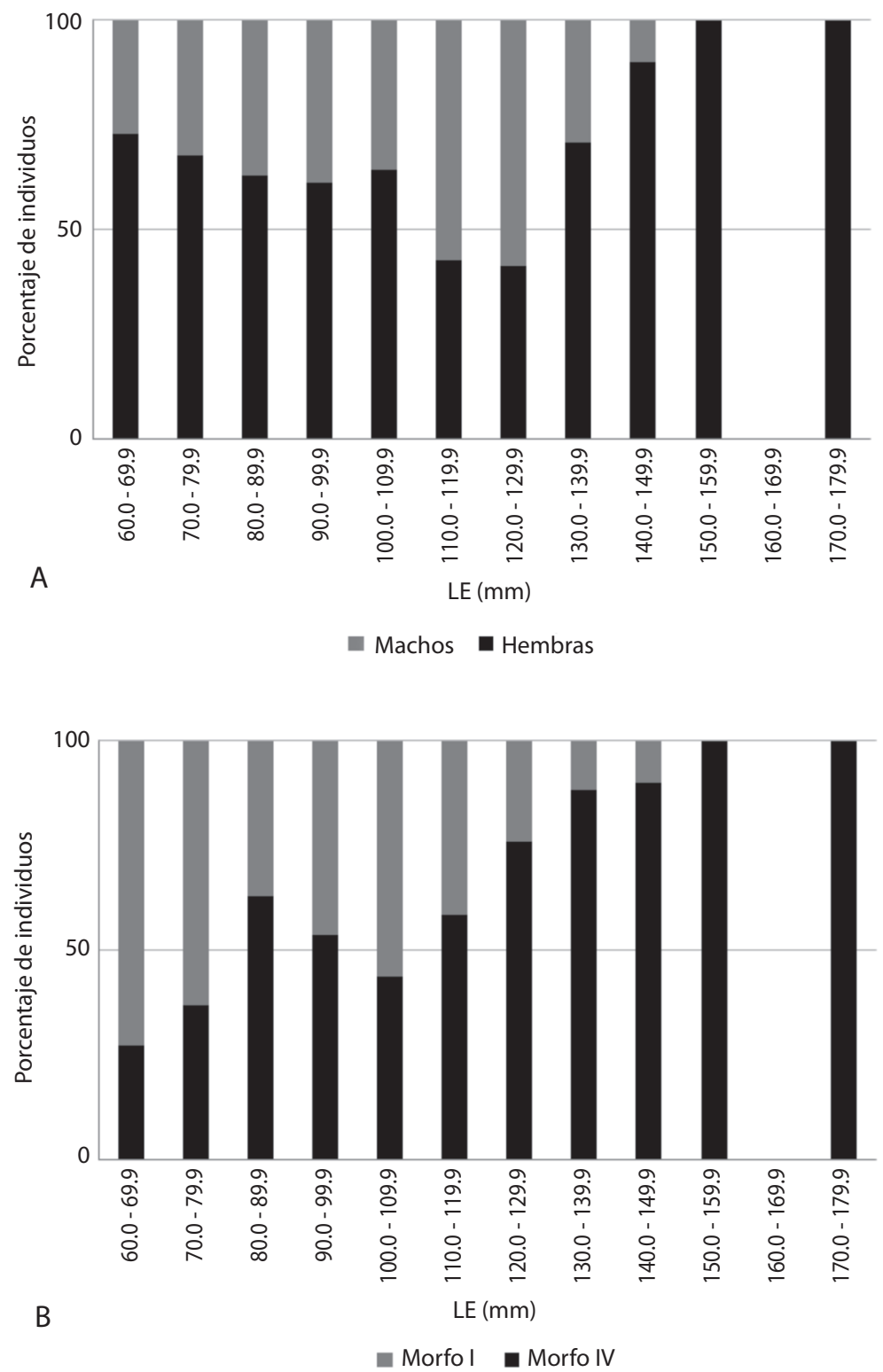

Fig. 1. (a) Frecuencia de hembras y machos, y (b) frecuencia de morfotipos orales de Saccodon dariensis en la parte media del río Guatapé, Oriente antioqueño, Colombia, según el tamaño $(\mathrm{N}=468)$. LE = longitud estándar.

Fig. 1. (a) Frequency of females and males, and (b) Frequency of oral morphotype of Saccodon dariensis in the Guatapé River mid-basin, Eastern Antioquia, Colombia, according to size $(\mathrm{N}=468)$. $\mathrm{LE}=$ Standard length. 
Índice gonadosomático: Los valores medios del índice gonadosomático (IGS) presentaron variaciones en cada muestreo y un comportamiento similar en ambos sexos. Los mayores valores de IGS se presentaron durante los períodos de transición de temporada seca a lluvia cuando empieza el aumento en el nivel de las aguas en marzo 2008 (Promedio IGS= 14.77 y 0.71 para hembras y machos respectivamente) y en septiembre 2011 (Promedio $\mathrm{IGS}=16.54$ y 0.65 para hembras y machos respectivamente) (Fig. 2).
Para ambos sexos, la escala de madurez sexual registró relación con los rangos de IGS. Los valores promedio más altos del IGS corresponden al estadio IV de individuos maduros con un valor promedio de IGS de 10.72 y 0.71 (hembras y machos respectivamente) (Cuadro 3). Los individuos en este estadio IV de maduración presentaron una talla entre los 67.7 y 174.0 $\mathrm{mm}$ de LE para hembras, y entre los $89.3 \mathrm{y}$ $149.4 \mathrm{~mm}$ de LE para los machos. Los registros medios del IGS presentaron un comportamiento similar en ambos morfotipos (Fig. 3).
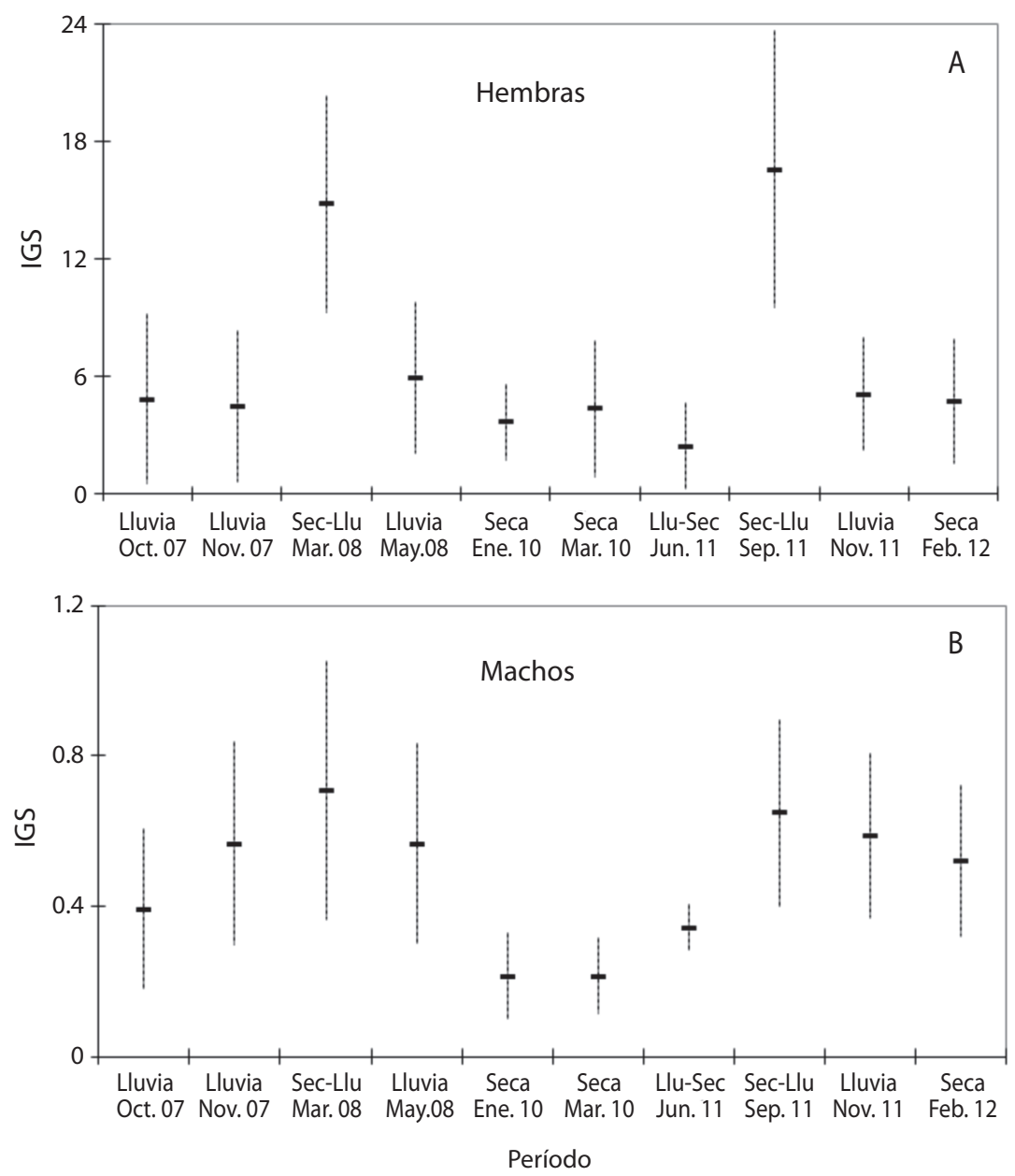

Fig. 2. Variación del Índice gonadosomático (IGS), los valores medios (+/-SD) para ambos sexos de Saccodon dariensis en la cuenca del río Guatapé, Oriente antioqueño, Colombia.

Fig. 2. Variation of the Gonadosomatic Index (GSI), the mean values (+/-SD) for both sexes of of Saccodon dariensis in the Guatapé River mid-basin, Eastern Antioquia, Colombia. 

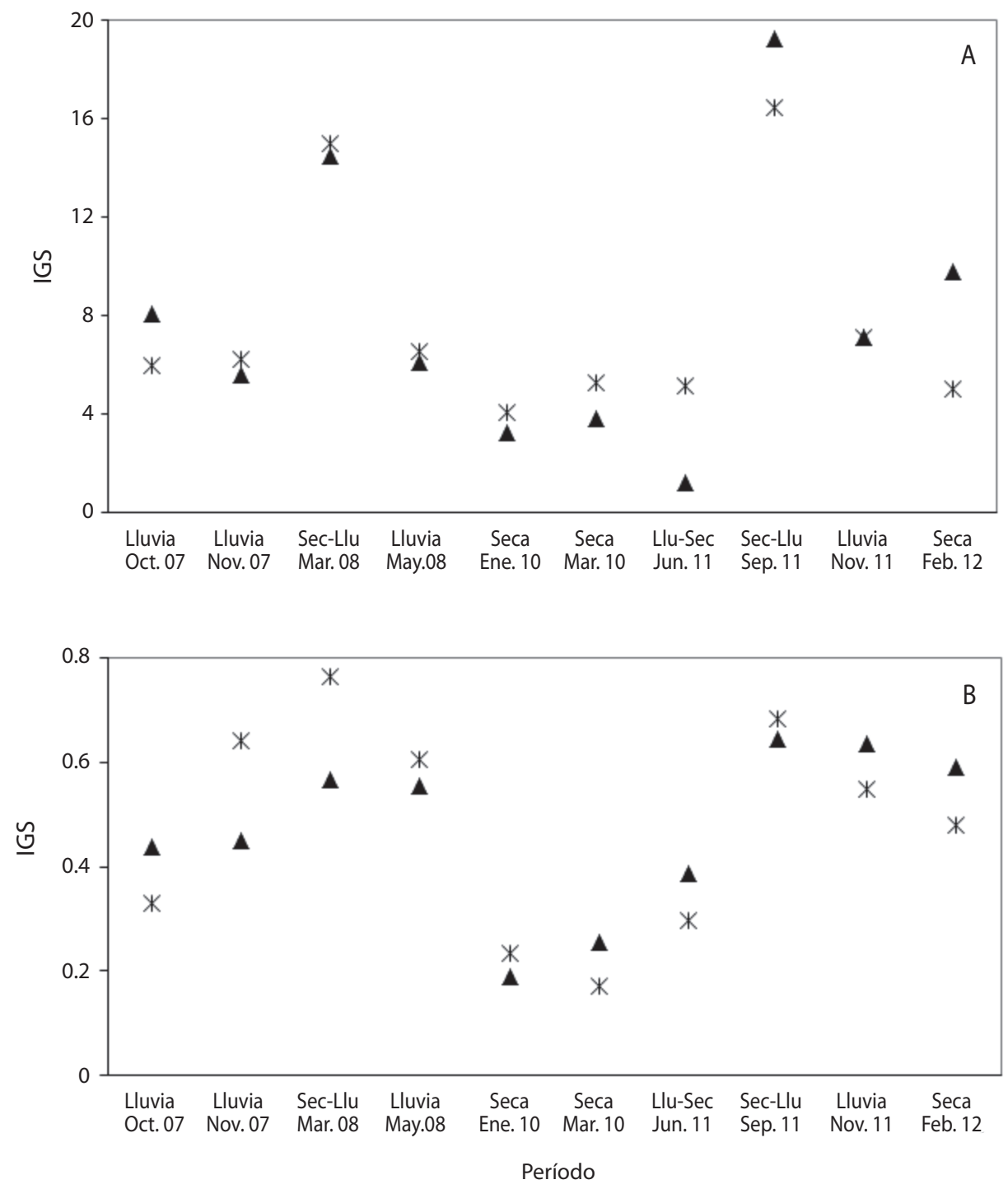

$\Delta$ Morfol $\quad$ M Morfo IV

Fig. 3. Variación del Índice gonadosomático (IGS) por morfotipos bucales para hembras (a) y machos (b) de Saccodon dariensis en la cuenca del río Guatapé, Oriente antioqueño, Colombia.

Fig. 3. Variation of the Gonadosomatic Index (GSI) by oral morphotype for females (a) and males (b) of Saccodon dariensis in the Guatapé River mid-basin, Eastern Antioquia, Colombia.

Factor de Condición (K) e Índice Hepatosomático: $\mathrm{El}$ factor de condición $(\mathrm{K})$ tuvo variaciones en cada muestreo, siendo más bajo durante los meses en que los valores del Índice gonadomático (IGS) fueron mayores, en los períodos de transición de temporada seca a lluvia en marzo $2008(\mathrm{~K}=0.73$ y 0.77 para hembras y machos, respectivamente) y en septiembre 2011 ( $\mathrm{K}=0.75$ y 0.82 para hembras y machos, respectivamente). Los mayores valores se presentaron en los períodos secos coincidiendo con menores valores de IGS (Fig. 4A). 
CUADRO 3

Escala de madurez sexual con relación al valor medio del índice gonadosomático (IGS)

y las características macroscópicas de cada estadio en el desarrollo de ovarios y testículos de Saccodon dariensis

TABLE 3

Sexual maturity scale with relation to the average value of the GSI, and the macroscopic characteristics of each stage in the development of Saccodon dariensis ovaries and testes

\begin{tabular}{|c|c|c|c|c|}
\hline Sexo & Estadio & IGS & $\mathrm{n}$ & Descripción macroscópica \\
\hline \multirow[t]{5}{*}{ Hembras } & I & $0.250 \pm 0.20$ & 7 & $\begin{array}{l}\text { Ovarios muy pequeños y acintados, de color crema o amarillo tenue, sin } \\
\text { productos sexuales. }\end{array}$ \\
\hline & II & $1.309 \pm 0.63$ & 65 & $\begin{array}{l}\text { Firmes, pero de tamaño pequeño. Ovarios rosados, translúcidos. Ovocitos } \\
\text { invisibles a simple vista. }\end{array}$ \\
\hline & III & $2.557 \pm 0.87$ & 38 & $\begin{array}{l}\text { Presentan un mayor tamaño, ovarios de color blanco o crema, se observan } \\
\text { los ovocitos de diferentes tamaños a simple vista. }\end{array}$ \\
\hline & IV & $10.724 \pm 6.02$ & 152 & $\begin{array}{l}\text { Los ovarios desarrollados, con un gran tamaño, ocupan la mayor o gran } \\
\text { parte de la cavidad abdominal. De color crema más fuerte que en el estado } \\
\text { anterior. Los ovocitos en el interior son de un tamaño uniforme a simple } \\
\text { vista. La gónada en general presenta una gran irrigación sanguínea. }\end{array}$ \\
\hline & V & $1.967 \pm 0.55$ & 6 & $\begin{array}{l}\text { Los ovarios están casi vacíos, flácidas, de color crema o rosado. Ovocitos } \\
\text { en el interior de diferente tamaño pueden ser visibles }\end{array}$ \\
\hline \multirow[t]{5}{*}{ Machos } & I & $0.162 \pm 0.10$ & 22 & $\begin{array}{l}\text { Testículos muy pequeños de forma plana y acintadas, bordes lisos, } \\
\text { generalmente muy blancos. }\end{array}$ \\
\hline & II & $0.260 \pm 0.09$ & 37 & $\begin{array}{l}\text { Los testículos y conductos de esperma aumentan en tamaño, son } \\
\text { acintados, de color blanco a crema y casi simétricos. }\end{array}$ \\
\hline & III & $0.500 \pm 0.11$ & 39 & $\begin{array}{l}\text { Las gónadas aumentan de tamaño, se ensanchan y presentan un color a } \\
\text { blanco a crema. }\end{array}$ \\
\hline & IV & $0.714 \pm 0.21$ & 93 & $\begin{array}{l}\text { Los testículos son desarrollados, de gran tamaño y generalmente están } \\
\text { llenos de esperma de color blanco. Ocupan gran parte de la cavidad } \\
\text { abdominal y son muy irrigados. }\end{array}$ \\
\hline & V & $0.222 \pm 0.17$ & 9 & $\begin{array}{l}\text { Las gónadas están flácidas, traslúcidas, poco irrigadas y con residuos de } \\
\text { esperma. }\end{array}$ \\
\hline
\end{tabular}

(I: Inmaduro, II: Reposo, III: En maduración, IV: Maduro o puesta y V: Desovado (hembras), espermiado (machos). n: número de individuos.

El IHS se comportó de una manera contraria al factor de condición $(\mathrm{K})$, siendo más alto en los períodos de transición de temporada seca a lluvia cuando los peces presentaron mayor desarrollo gonadal y valor de IGS (Fig. 4B). En ambos sexos, las variaciones de IHS y $\mathrm{K}$ fueron muy perceptibles, lo que sugiere que tienen una alta relación con los procesos de maduración.

Se presentó mayor proporción de estadios de madurez sexual en fase IV (Maduro) y $\mathrm{V}$ (Desovado en hembras o espermiado en machos) durante los períodos de seca a lluvia y de lluvia tanto para hembras como para machos. Durante los períodos secos se observó mayor proporción de estadios de madurez sexual en fases I (Inmaduro), II (reposo) y III (en maduración) (Fig. 5). Se observó la presencia en machos adultos de tubérculos nupciales durante la época reproductiva.

Talla de madurez sexual: Los ejemplares maduros más pequeños se presentaron a una talla de 67.7 y $89.3 \mathrm{~mm}$ de LE, para hembras y machos, respectivamente. La función logística mostró que la talla media de madurez sexual $\left(\mathrm{L}_{50}\right)$ fue de $88.8 \mathrm{~mm}$ de LE para las hembras y $109.3 \mathrm{~mm}$ para los machos (Fig. 6A). El análisis por morfotipos de la talla media de madurez sexual muestra un comportamiento similar en ambos morfotipos (Fig. 6B). 

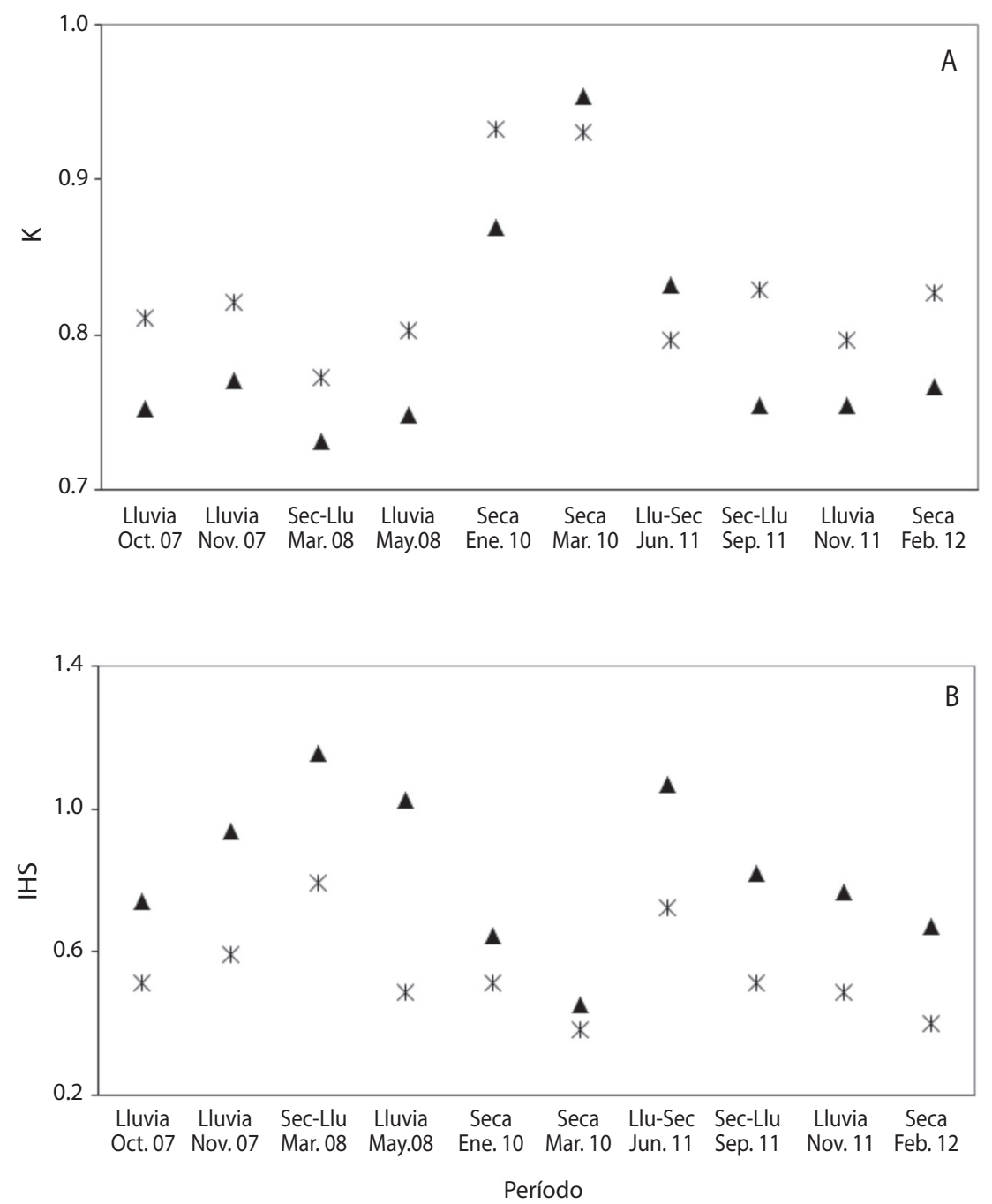

A Hembras $*$ Machos

Fig. 4. Factor de condición (K) e índice hepatosomático (IHS) para hembras y machos de Saccodon dariensis en la cuenca del río Guatapé, Oriente antioqueño, Colombia.

Fig. 4. Condition factor (K) and Hepatosomatic Index (HIS) by females and males of Saccodon dariensis in the Guatapé River mid-basin, Eastern Antioquia, Colombia.

Fecundidad: El número medio de ovocitos presentes en las gónadas examinadas de 63 hembras maduras de Saccodon dariensis capturadas durante el período reproductivo fue $8309(\mathrm{DE}=9021)$ con un rango entre 113739303. El valor máximo de 39303 ovocitos se presentó a una talla de $126.0 \mathrm{~mm} \mathrm{LE}$, y el valor mínimo de 1137 ovocitos a una de 73.2 cm LE. La fecundidad absoluta aumentó con la longitud estándar de acuerdo a la ecuación $\mathrm{F}=$ $588.65 \mathrm{LE}^{3.25}\left(\mathrm{R}^{2}=0.68\right)$. La ecuación que describe la relación entre la fecundidad absoluta $\mathrm{F}$ y peso eviscerado es $\mathrm{F}=588.65 \mathrm{PEv}^{0.952}\left(\mathrm{R}^{2}=\right.$ $0.66)$ y con el peso de las gónadas fue $F=4593$ $\mathrm{Pg}^{0.911}\left(\mathrm{R}^{2}=0.89\right)$. La fecundidad relativa al peso total fue de 439 ovocitos/g $(\mathrm{DE}=212.3)$, 

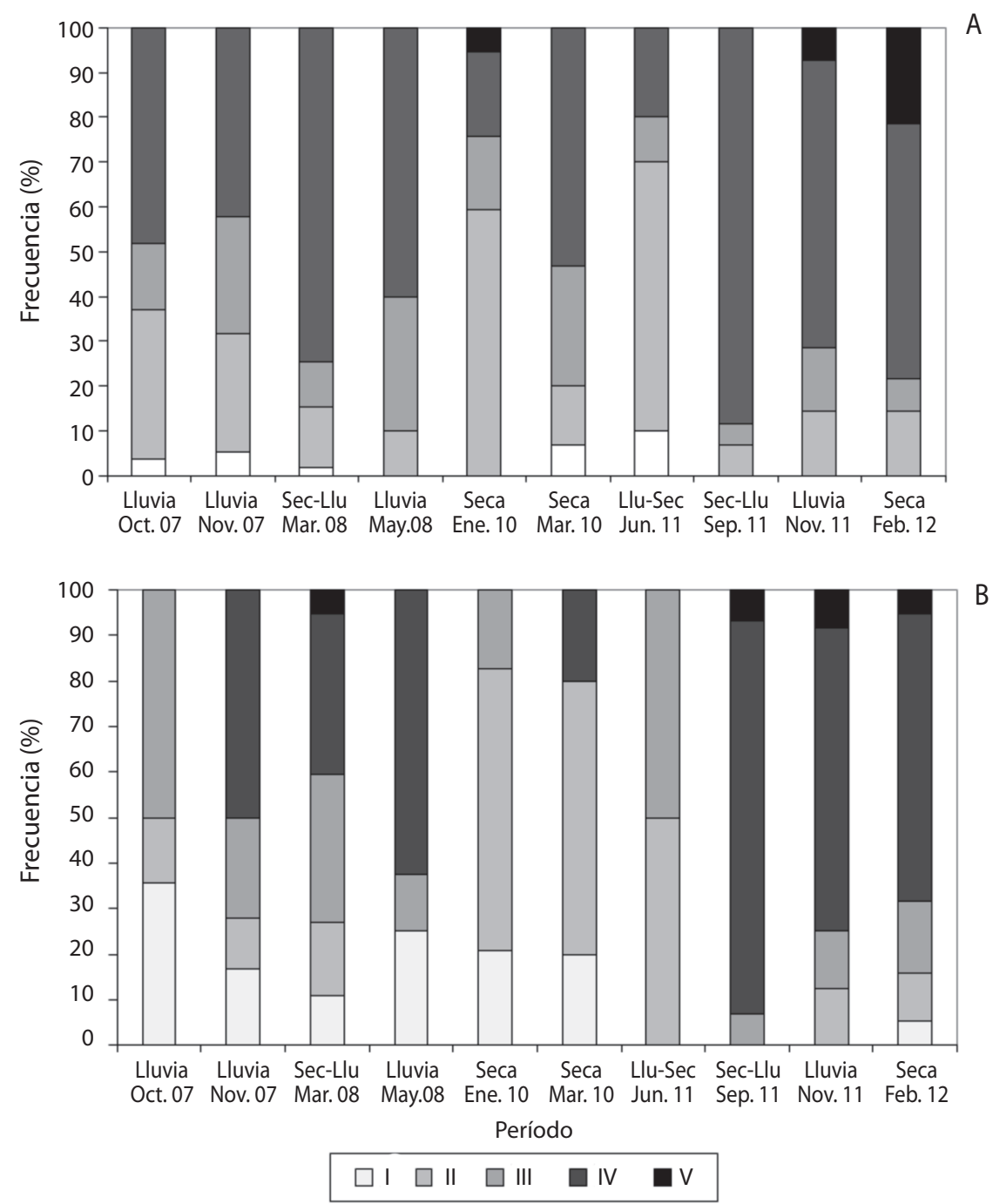

Fig. 5. Proporción de estadios de madurez sexual por período de hembras (a) y machos (b) de Saccodon dariensis en la cuenca del río Guatapé, Oriente antioqueño, Colombia.

Fig. 5. Proportion of sexual maturity stages for each period of females (a) and males (b) of Saccodon dariensis in the Guatapé River mid-basin, Eastern Antioquia, Colombia.

al peso eviscerado fue de 590.1 ovocitos/g $(\mathrm{DE}=294.5)$ y al peso de las gónadas fue de 4 676.6 ovocitos/g $(\mathrm{DE}=1$ 590.3). El diámetro de los ovocitos varió entre 0.30 y $0.80 \mathrm{~mm}$ de diámetro, con un pico de la frecuencia máxima de ovocitos de diámetros entre 0.50 y $0.60 \mathrm{~mm}$. El diámetro medio fue de $0.54 \mathrm{~mm}(\mathrm{DE}=0.07)$. No se observaron diferentes estados de desarrollo en los ovocitos que sugirieran desoves parciales. No hubo diferencia en el diámetro medio de los ovocitos presentes en las gónadas en relación con la longitud estándar (Correlación de Pearson $=-0.03 ; \mathrm{P}=0.78$ ).

\section{DISCUSIÓN}

Saccodon dariensis presentó una longitud máxima de 174.0 y $149.4 \mathrm{~mm}$ LE para hembras 

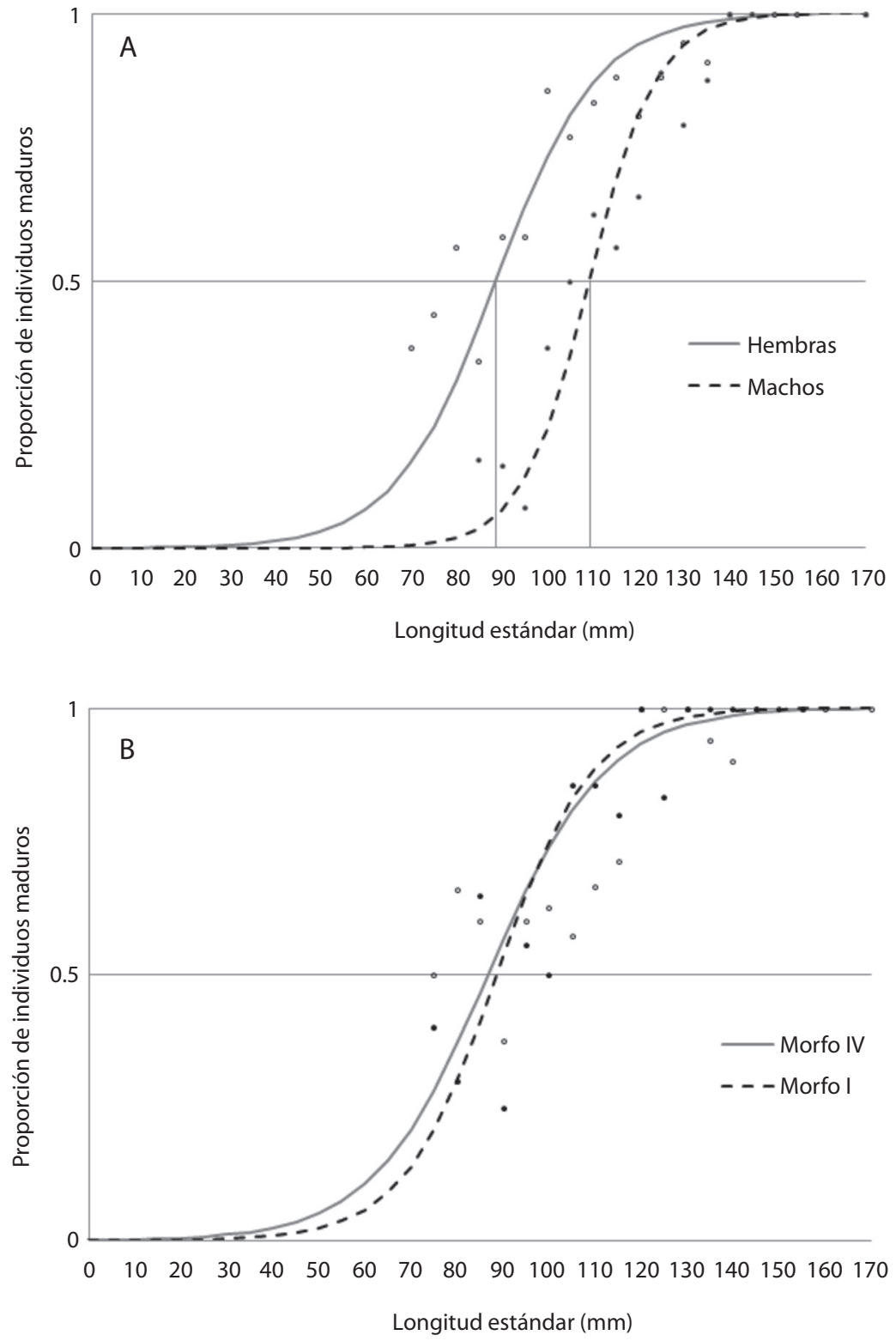

Fig. 6. (a) Curva de madurez sexual y talla media de madurez sexual $\left(\mathrm{L}_{50}\right)$ para hembras y machos, y (b) por morfotipos bucales de Saccodon dariensis en la cuenca media del río Guatapé.

Fig. 6. (a) Curve of sexual maturity and the mean size of sexual maturity $\left(\mathrm{L}_{50}\right)$ for females and males, and (b) by oral morphotype of Saccodon dariensis in the Guatapé River mid-basin.

y machos, respectivamente, lo cual amplía el valor máximo de longitud reportado por Pavanelli (2003) de $127 \mathrm{~mm}$ de LT para esta especie. Este valor es superior al encontrado para otras especies de la familia como Apareiodon affinis
(151 mm LT), A. piracicabae (165 mm LT) y Parodon nasus (117 mm LT) (Graça \& Pavanelli, 2007)

La proporción de sexos se presentó a favor de las hembras (1.00: 1.34), y cambió con la 
longitud, siendo las hembras más frecuentes en tamaños menores a los $109.9 \mathrm{~mm}$ y mayores a $130 \mathrm{~mm}$ de LE, mientras que los machos fueron más frecuentes en el intervalo entre los 110 y los $129.9 \mathrm{~mm}$ de LE. La proporción de sexos en peces varía a lo largo de su ciclo de vida, generalmente en una población es de 1:1 (Thomé, Bazzoli, Rizzo, Santos, \& Ratton, 2005), pero esta puede variar por diferentes eventos de mortalidad, tasas de crecimiento y entre distintas localidades. De manera comparada, para Parodon magdalenensis en el río La Miel ha sido reportada una proporción favorable a los machos (1.00:0.88) (Pareja \& Jiménez-Segura, 2012).

Igualmente, se encontró una proporción de morfotipos desequilibrada a favor del morfotipo IV que predominó en todo el estudio, al igual que para la mayoría de tallas. Los valores medios de LE y PT no fueron significativamente diferentes entre sexos, pero si entre morfotipos bucales, lo que indica una mayor adecuación del morfotipo IV. Esto podría explicarse por tasas de crecimiento diferencial debido a variaciones en la dieta entre morfotipos que les confiere acceso diferencial a los ítems que constituyen su dieta y diferentes influencias a nivel nutricional (Restrepo-Gómez \& Mancera-Rodríguez, 2014).

Restrepo-Escobar, Rangel-Medrano, Mancera-Rodríguez y Márquez (2015) encontraron que los morfotipos de boca I y IV de $S$. dariensis presentan diferencias en el tamaño y forma del cuerpo y, que no existen diferencias significativas en el tamaño entre los sexos para el morfotipo I, mientras que si las hay para el morfotipo IV donde las hembras mostraron un tamaño promedio mayor que los machos, lo cual puede explicar el hecho de que por encima de los $150 \mathrm{~mm}$ de LE solo hayan sido capturadas hembras de este morfotipo.

Esta especie muestra un crecimiento alométrico positivo más marcado en los machos, que indica una ganancia en peso en relación con la longitud estándar. En otras especies de Parodontidae ha sido reportado crecimiento con tendencia a la alometría positiva en Parodon magdalenensis en el río La Miel Pareja y
Jiménez-Segura (2012), así como en machos de $P$. nasus $(\mathrm{b}=3.17)$ y A. ibitiensis $(\mathrm{b}=3.12)$ y hembras de Apareiodon affinis $(\mathrm{b}=3.26)$ en Brasil (Barbieri et al., 1985).

El incremento en los valores de IGS está asociado con la maduración, siendo para las hembras de $S$. dariensis mucho más altos (aproximadamente 10-20 veces) que los registrados en los machos, y los ovarios maduros fueron mayores que los testículos maduros. Variaciones similares han sido reportadas para Apareiodon affinis en Brasil (Fonseca-Ratton et al., 2003). El período de mayor desarrollo gonadal de $S$. dariensis en las quebradas afluentes del río Guatapé se produce durante los períodos de transición de temporada seca a lluviosa, cuando empieza a subir el nivel de las aguas. Esto coincide con lo reportado para la especie en la cuenca alta del río Cauca, en donde se reproduce al comienzo de las lluvias entre octubre y noviembre (Usma-Oviedo et al., 2013). Para otras especies de la familia en Brasil como Apareiodon piracicabae, A. ibitiensis y Parodon nasus se ha registrado que se reproducen en la temporada de lluvias (Barbieri et al., 1983; Barbieri et al., 1985; Azevedo et al., 1988a, b; Barbieri \& Barbieri, 1989) y para Apareiodon affinis Bialetzki et al. (1998) menciona que los cambios ambientales como el comienzo de la subida del nivel del río, probablemente influyen en la reproducción de la especie. Godoy (1975) indicó que las especies de esta familia, en el suroriente de Brasil, ascienden por el río para desovar durante la temporada de lluvias. La sincronía entre el período reproductivo y las inundaciones tiene por objeto garantizar cualitativa y cuantitativamente la máxima disponibilidad de alimentos en las primeras etapas de desarrollo, proporcionan un rápido crecimiento y superan las etapas vulnerables a la depredación intensa (Welcomme, 1979).

En ninguno de los períodos de muestreo se encontraron alevines y juveniles de la especie, menores a $65 \mathrm{~mm}$ de LE, en las dos quebradas, y se encontró un bajo porcentaje de ejemplares con gónadas en estado posterior al desove; esto permite inferir que Saccodon dariensis realiza 
desplazamientos con fines reproductivos desde las quebradas al cauce principal del río donde realiza el desove y luego los juveniles se desplazan nuevamente hacia las quebradas donde crecen. Al respecto, Usma-Oviedo et al. (2013) encontraron que las etapas juveniles de $S$. dariensis presentan migraciones cortas, desde el cauce principal del Cauca, hacia los tributarios menores del piedemonte, en donde crecen y se establecen como adultos.

A diferencia de los prolongados períodos reproductivos y la ocurrencia de múltiples desoves fraccionados encontrados en Brasil para Apareiodon affinis, A. piracicabae, A. ibitiensis y Parodon nasus (Nomura et al., 1978; Barbieri et al., 1983; Barbieri et al., 1985; Fonseca-Ratton et al., 2003; Gomiero \& Braga, 2007), para Saccodon dariensis se observó estados de desarrollo similares en los ovocitos, que sugieren desoves masivos concentrados en cortos períodos de tiempo. Esto se puede deber a las diferentes condiciones ambientales en que se encuentra la especie en zonas de montaña con un régimen de lluvias bimodal con dos períodos marcados de lluvia y dos períodos secos al año.

La variación en el factor de condición es opuesta a los valores del IGS, con valores más altos durante los períodos secos, que suponen un buen estado de nutrición del pez como preparación para el evento reproductivo y los más bajos durante el período de reproducción, que indican, que esta especie acumula reservas de energía corporal que son utilizadas durante la maduración gonadal y el desove. Esto coincide con lo encontrado por Barbieri et al. (1985) para Apareiodon affinis, A. ibitiensis y Parodon nasus, y por Fonseca-Ratton et al. (2003) en hembras de $A$. affinis, siendo el factor de condición un indicador ampliamente utilizado de la condición fisiológica de los peces (Vazzoler, 1996).

Los valores de IHS en hembras normalmente descienden durante los estadios de maduración y maduros debido a la transferencia de sustancias hepáticas durante la vitelogénesis (Selman \& Wallace, 1989). Sin embargo, para $S$. dariensis no fueron registrados valores bajos durante los períodos de mayor madurez gonadal que permitan correlacionar la transferencia de sustancias hepáticas durante la maduración del ovario. Fonseca-Ratton et al. (2003) encontró resultados similares para $A$. affinis en el embalse de Furnas en Minas Gerais, Brasil.

Los tamaños de primera maduración sexual se presentaron a una talla de $67.7 \mathrm{y}$ $89.3 \mathrm{~mm}$ de LE para hembras y machos respectivamente. Que son cercanas a las tallas de ejemplares maduros más pequeños reportadas para Apareiodon affinis (80 $\mathrm{mm}$ de LT), A. ibitiensis (69 mm de LT) y Parodon nasus (75 mm de LT) (Barbieri et al., 1985). Suzuki et al. (2004) registra para hembras de A. affinis un tamaño de primera maduración sexual de $73 \mathrm{~mm}$ de LT, menor que para machos que es de $79 \mathrm{~mm}$ de LT.

El tamaño al $50 \%$ de madurez sexual $\left(\mathrm{L}_{50}\right)$ fue de $88.8 \mathrm{~mm}$ de LE para hembras y 109.3 $\mathrm{mm}$ de LE para los machos. Las variaciones en longitud de maduración sexual entre hembras y machos son atribuibles al dimorfismo sexual, considerando que las variaciones que se encuentran en hábitats están asociados a la disponibilidad de alimentos y el resultado de tasas diferenciales de crecimiento (Nikolsky, 1963). Las hembras alcanzan la primera madurez sexual más rápido que los machos (tallas inferiores), lo que tiene fuertes implicaciones a nivel poblacional en sus estrategias de vida, y puede deberse a una estrategia para la eliminación de competencia interespecífica, siendo una respuesta que permite a las hembras tener más posibilidades de dejar descendencia, debido a la rápida maduración (Granados, 1996).

Los valores de fecundidad absoluta de las hembras de $S$. dariensis con una media de 8309 ovocitos y de fecundidad relativa media de 439 ovocitos/g son similares a los reportados para Parodon magdalenensis en la cuenca del río Magdalena con valores de 9244 ovocitos y 535 ovocitos/g (Pareja \& Jiménez-Segura, 2012). Para Brasil, se han reportado valores de fecundidad absoluta de las hembras de Apareiodon affinis (6432 ovocitos), A. ibitiensis (2245 ovocitos) y Parodon nasus (20 455) (Barbieri et al., 1985). Igualmente, la fecundidad estimada 
para $S$. dariensis fue alta comparada con otras especies de peces de alta montaña neotropical (Román-Valencia, Hernández, \& Samudio, 2007; García-Alzate \& Román-Valencia, 2008; Román-Valencia, Ruiz-C, \& Giraldo, 2008). Esta alta fecundidad puede ser una estrategia de historia de vida, en la que hay una respuesta a diferentes factores de selección como presencia de depredadores, estrés por disponibilidad de recursos, estocasticidad ambiental, altas tasas de mortalidad y de capturas (Caswell, Naiman, \& Morin, 1984), y el no cuidado parental que ha sido reportado para otras especies de parodontidos (Graça \& Pavanelli, 2007).

Se observó que las hembras más grandes produjeron más huevos por unidad de peso corporal y la fecundidad absoluta aumentó con la longitud estándar. La fecundidad relativa depende de la longitud del pez, aunque esta puede ser influenciada por otros factores tales como el medio ambiente y la condición de bienestar de los individuos (Rait \& Hall, 1967). El diámetro de los ovocitos varió entre 0.30 y $0.80 \mathrm{~mm}$ de diámetro y un diámetro medio de $0.54 \mathrm{~mm}$. Así, los ovocitos maduros tienen un rango de tamaño similar al registrado para Parodon magdalenensis, con un diámetro entre 0.20 y $0.91 \mathrm{~mm}$ y un valor medio de $0.59 \mathrm{~mm}$ (Pareja \& Jiménez-Segura, 2012). Para otras especies de Parodóntidos no se han reportado valores. Se observó la presencia de tubérculos nupciales en los machos adultos durante la época reproductiva confirmando el dimorfismo sexual señalado por Wiley and Collette (1970). Estas estructuras probablemente evolucionaron originalmente para permitir a los individuos mantener un estrecho contacto durante el desove para asegurar la fertilización de los huevos, lo cual es particularmente importante en los peces que desovan en aguas rápidas (Wiley \& Collette, 1970). Igualmente, Londoño-Burbano \& Román-Valencia (2010) mencionan la presencia de dimorfismo sexual, aunque no observaron tubérculos nupciales en los ejemplares evaluados.

La coexistencia de los dos morfotipos, la reproducción externa y la ocurrencia simultánea de los picos reproductivos encontrada en este estudio, no proporcionan apoyo a un posible aislamiento reproductivo espacial o temporal de los morfotipos. Sin embargo, deben estudiarse otras posibles explicaciones como el polifenismo, en el que múltiples fenotipos discretos pueden surgir de un solo genotipo como resultado de diferentes condiciones ambientales, o diferencias genéticas resultantes de especiación críptica o el apareamiento selectivo pre o postcigótico.

La especie presenta una estrategia reproductiva estacional de acuerdo a las estrategias reproductivas propuestas por Winemiller (1989) con características como reproducción estacional y cíclica, sin cuidado parental, y alta fecundidad. Similar a lo propuesto por Gomiero y Braga (2007) para Apareiodon piracicabae, A. ibitiensis y Parodon nasus.

Las características reproductivas descritas proporcionan elementos para la conservación de la especie, siendo primordial la protección de cuerpos hídricos estudiados que son áreas de importancia para el crecimiento y desarrollo de la especie hacia etapas adultas y en las que se observó que un alto porcentaje de los individuos están por debajo de la talla media de madurez sexual, principalmente en la quebrada el Cardal. Estos cuerpos hídricos se han visto fuertemente afectados por actividades antrópicas ganaderas, agrícolas y la descarga de aguas residuales, siendo prioritario que se establezcan medidas de protección, en especial en la quebrada el Cardal, en la cual se han encontrado dos de las tres especies de peces carácidos del genero Hemibrycon que han sido recientemente descritas (Román-Valencia, Ruiz-C, Taphorn, Mancera-Rodríguez, \& García-Alzate, 2013).

\section{AGRADECIMIENTOS}

A la Dirección de Investigación Medellín-DIME de la Universidad Nacional de Colombia, quien financió el Proyecto de Investigación, en la Convocatoria de proyectos para estudiantes de pregrado año 2009. A la empresa ISAGEN S.A. E.S.P., a la Corporación Autónoma Regional de los Ríos Negro y NareCORNARE y a la Universidad Nacional de 
Colombia por la financiación del proyecto 20101009235 "Estudio de la biología, ecología y diversidad genética de las poblaciones naturales de sabaleta Brycon henni en las cuencas de los ríos Nare y Guatapé, Antioquia", dentro del cual fue recolectado el material biológico base en este estudio.

\section{RESUMEN}

Colombia posee una alta diversidad de peces de montaña dentro de la región Andina, con alto número de endemismos, por lo que el conocimiento de su biología reproductiva es necesario para su protección y conservación. Se estudió la biología reproductiva de Saccodon dariensis en las quebradas Peñoles y El Cardal, parte media del río Guatapé, cuenca del río Magdalena, Colombia, para determinar si existen diferencias reproductivas entre individuos con diferente polimorfismo bucal (morfo I vs morfo IV, definidos por Roberts, 1974) que permitan definir un posible aislamiento reproductivo espacial o temporal. Se realizaron diez muestreos de campo entre octubre 2007 y febrero 2012, en períodos de lluvia (octubre y noviembre 2007, mayo 2008 y noviembre 2011) seco (enero y marzo 2010, febrero 2012), transición de lluvia a seca (junio 2011) y transición de seca a lluvia (marzo 2008 y septiembre 2011). Se efectuaron capturas de individuos por medio de equipo de electropesca y de atarraya con ojo de malla de $1 \mathrm{~cm}$. Se analizaron 468 especímenes, de los cuales 268 fueron hembras y 200 machos. La talla media de captura para el total de individuos estudiados fue de 109.6 mm de LE (65.5-174.0 mm), siendo para las hembras de $108.0(67.7-174.0) \mathrm{mm}$ de LE y para los machos de $111.9 \mathrm{~mm}$ de LE (65.5-149.4). Las hembras predominaron en las capturas, y la proporción de sexos de 1.0:1.34 se desvió significativamente de la distribución teórica 1:1. Igualmente el morfotipo IV predominó en las capturas y la proporción de morfotipos fue de 1.0:1.48. Con base en la evolución mensual del índice gonadosomático (IGS) y la proporción de individuos maduros, la temporada de desove se produce durante los períodos de transición de temporada seca a lluvia cuando empieza a subir el nivel de las aguas. Los menores valores del factor de condición que coinciden con los valores máximos del IGS indican que esta especie acumula reservas de energía corporal que son utilizadas durante la maduración gonadal y el desove. La talla media de madurez sexual $\left(\mathrm{L}_{50}\right)$ para los individuos de $S$ dariensis se alcanzó en las hembras a los $88.8 \mathrm{~mm}$ de LE y en los machos a los $109.3 \mathrm{~mm}$ de LE. La fecundidad fluctuó entre 1137 y 39303 ovocitos (media $=8309 ; \mathrm{DE}=9021)$ y la fecundidad relativa osciló entre 144 y 1131 ovocitos / g de peso total (media $=439 \pm 212)$. El diámetro de los oocitos fue de $0.54 \mathrm{~mm}(\mathrm{SD}=0.07)$. No se observó desarrollo diferente en ovocitos, lo que sugiere un desove masivo. La coexistencia de los dos morfotipos, la reproducción externa y la ocurrencia simultánea de los picos reproductivos encontrada en este estudio, no proporcionan apoyo a un posible aislamiento reproductivo espacial o temporal de los morfotipos.

Palabras clave: conservación, aspectos reproductivos, pez tropical de aguadulce, talla media de madurez sexual.

\section{REFERENCIAS}

Anderson, R. O. \& Gutreuter, S. J. (1983). Length, weight, and associated structural indices. In L. A. Nielsen \& D. L. Johnson (Eds.), Fisheries Techniques (pp. 283-300). Bethesda, Maryland: American Fisheries Society.

Azevedo, C. O., Barbieri, M. C., \& Barbieri, G. (1988a) Ciclo reprodutivo de Parodon tortuosus (Eigenmann $\&$ Norris, 1900) do rio Passa-Cinco, Ipeuna-SP. I. Estádios de maturação dos testículos. Época de reprodução. Revista Brasileira de Biología, 48, 565-569.

Azevedo, C. O., Barbieri, M. C., \& Barbieri, G. (1988b) Ciclo reprodutivo de Parodon tortuosus (Eigenmann \& Norris, 1900) do rio Passa-Cinco, Ipeuna-SP. II. Estádios de maturação do ovário. Época de reprodução. Revista Brasileira de Biología, 48, 571-575.

Barbieri, G., \& Barbieri, M. C. (1989) Growth and first sexual maturation size of Parodon tortuosus Eigenmann \& Norris, 1900 from Passa Cinco river (Ipeuna, São Paulo State, Brazil) (Osteichthyes, Parodontidae). Naturalia, 14, 45-54

Barbieri, G., Verani, J. R., Pereira, J. A., Barbieri, M. C., Peret, A. C., \& Marins, M. A. (1985) Curva de maturação e fator de condição de Apareiodon affinis (Steindachner, 1879), Apareiodon ibitensis (Campos, 1944) e Parodon tortuosus (Eigenman \& Norris, 1900) do rio Passa Cinco, Ipeúna-S.P. (Cypriniformes, Parodontidae). Ciência e Cultura, 37, 1178-1183.

Barbieri, G., Verani, J. R., \& Barbieri, M. C. (1983). Análise do comportamento reprodutivo das espécies Apareiodon affinis (Steindachner, 1879), Apareiodon ibitiensis Campos, 1944 e Parodon tortuosus, Eingenmann \& Norris, 1900 do rio Passa Cinco, Ipeúna, SP (Pisces, Parodontidae). In G. Strixino, L. C. A. Bertollo, M. M. Dias Filho e M. H. A. O. Sousa (Eds.), Anais do III Seminário Regional de Ecologia (pp. 189-192). São Carlos: Universidade Federal de São Carlos.

Bialetzki, A., Sanches, P. V., Baumgartner, G., \& Nakatani, K. (1998). Caracterizacão morfológica e distribuicão temporal de larvas e juvenis de Apareiodon affinis (Steindachner) (Osteichthyes, Parodontidae) no alto rio Paraná, Paraná. Revista Brasileira de Zoología, 15, 1037-1047. 
Caswell, H., Naiman, R. J., \& Morin, R. (1984). Evaluating the consequences of reproduction in complex salmonid life cycles. Aquaculture, 43, 123-134.

Fonseca-Ratton, T., Bazzoli, N., \& Bastos-Santos, G. (2003). Reproductive biology of Apareiodon affinis (Pisces: Parodontidae) in the Furnas Reservoir, Minas Gerais, Brazil. Journal of Applied Ichthyology, 19, 387-390.

García-Alzate, C. A. \& Román-Valencia, C. (2008). Biología alimentaria y reproductiva de Hyphessobrycon poecilioides (Pisces: Characidae) en la cuenca del rio La Vieja, Alto Cauca, Colombia. Revista del Museo Argentino de Ciencias Naturales, 10(1), 17-27.

Godoy, M. P. (1975). Peixes do Brasil Sub-ordem Characoidei- Bacia do rio Mogi Guassu. Piracicaba. Franciscana, 3, 400-627.

Gomiero, L. M., \& Braga, F. M. S. (2007). Reproduction of a fish assemblage in the state of São Paulo, southeastern Brazil. Brazilian Journal of Biology, 67(2), 283-292.

Granados, L. C. (1996). Ecología de Peces. España: Secretariado publicaciones Universidad de Sevilla.

Graça, W. J. D., \& Pavanelli, C. S. (2007). Peixes da planice de inundação do alto do rio Paraná e áreas adjacentes. Maringá: EDUEM.

Hammer, Ø., Harper, D. A. T., \& Ryan, P. D. (2001). PAST: Paleontological Statistics Software Package for Education and Data Analysis. Palaeontología Electrónica [Computer program]. Retrived from http://palaeoelectronica. org/2001_1/past/issue1_01.htm.

Holden, M. J. \& Raitt, D. F. S. (1975). Manual de ciencia pesquera, Parte II. Métodos para investigar los recursos y su aplicación (Documento técnico de pesca No. 115). FAO.

Holdridge, L. R. (1967). Life zone ecology. Costa Rica: Centro Científico Tropical.

Jaramillo-Villa, U., Maldonado-Ocampo, J. A., \& BogotáGregory, J. D. (2008). Peces del Oriente de Antioquia, Colombia. Biota Colombiana, 9(2), 279-293.

Londoño-Burbano, A. \& Román-Valencia, C. (2010). Redescripción de Parodon caliensis y Saccodon dariensis (Characiformes: Parodontidae). Revista de Biología Tropical, 58(3), 813-826.

Maldonado-Ocampo, J. A., Vari, R. P., \& Usma-Oviedo, J. S. (2008). Checklist of the Freshwater Fishes of Colombia. Biota Colombiana, 9(2), 143-237.

Nikolsky, G. V. (1963). The Ecology of Fishes. London: Academic Press.

Nomura, H. (1979). Caracteres merísticos e dados biológicos sobre o canivete, Parodon tortuosus Eigenmann
\& Morris, 1990 do rio Mogi Guaçu, São Paulo (Osteichthyes, Parodontidae). Revista Brasileira de Biología, 39(2), 451-456.

Nomura, H., Ferreira, M., \& Hayashi, C. (1978) Caracteres merísticos e dados biológicos sobre o canivete, Apareiodon affinis (Steindachner, 1879) do rio Mogi Guaçu, São Paulo (Osteichthyes, Parodontidae). Revista Brasileira de Biología, 38(4), 745-752.

Oldani, N. O. 1977. Identificación y morfología de larvas de Apareiodon affinis (Steindachner) (Pisces, Parodontidae). Physis, 37(93), 133-140.

Pareja, M. \& Jiménez-Segura, L. F. (2012). Algunos aspectos de la biología reproductiva de Parodon magdalenensis (Characiformes: Parodontidae). Memorias IX Seminario Colombiano de Limnología. Actualidades Biológicas, 34(96), 168.

Pavanelli, C. S. (2003). Parodontidae (Parodontids). In R. E. Reis, S. O. Kullander, \& C. J. Ferraris, Jr. (Eds.), Checklist of the Freshwater Fishes of South and Central America (pp. 46-50). Porto Alegre, Brasil: EDIPUCRS

Rait, F. S. \& Hall, W. B. (1967). On the fecundity of the redfish, Sebastes marinus (L.). Journal du Conseil / Conseil Permanent International pour l'Exploration de la Mer, 31, 237-245.

Restrepo-Escobar, N., Rangel-Medrano, J. D., ManceraRodríguez, N. J., \& Márquez, E. J. (2015). Molecular and morphometric characterization of two dental morphs of Saccodon dariensis (Pisces: Parodontidae). In Memorias XXI Encontro Brasileiro de Ictiologia, 2015, Recife, Brasil. p.787.

Restrepo-Gómez, A. M., \& Mancera-Rodríguez, N. J. (2014). Trophic ecology of Saccodon dariensis (Pisces: Parodontidae) in Guatapé River tributaries, Magdalena River Basin, Colombia. Journal MVZ Córdoba, 19(1), 3930-3943.

Roberts, T. (1974). Dental polymorphism and systematics in Saccodon, a neotropical genus of freshwater fishes (Parodontidae, Characoidei). Journal of Zoology London, 173, 303-321.

Rodríguez, N., Armenteras, D., Morales, M., \& Romero, M. (2004). Ecosistemas de los Andes colombianos. Bogota: Instituto de investigación de recursos Biológicos Alexander von Humboldt.

Román-Valencia, C., Hernández, J. H., \& Samudio, H. F. (2007). Sobre ecología de Characidium caucanum (Pisces: Crenuchidae) en el alto río Cauca, Colombia. Revista de la Asociación Colombiana de Ictiologos Dahlia, 9, 33-42.

Román-Valencia, C., Ruiz-C, R. I., \& Giraldo, A. (2008). Dieta y reproducción de dos especies sintópicas: Hemibrycon boquiae y Bryconamericus caucanus (Pisces: Characidae) en la quebrada Boquía, rio 
Quindío, Alto Cauca, Colombia. 2008. Revista del Museo Argentino de Ciencias Naturales, 10(1), 55-62.

Román-Valencia, C., Ruiz-C., R., Taphorn, D. C., Mancera-Rodríguez, N. J., \& García-Alzate, C. A. (2013). Three new species of Hemibrycon (Characiformes: Characidae) from the Magdalena River Basin, Colombia. Revista de Biología Tropical, 61, 1365-1387.

Rondineli, G. R., \& Braga, F. M. S. (2010). Reproduction of the fish community of Passa Cinco Stream, Corumbataí River sub-basin, São Paulo State, Southeastern Brazil. Brazilian Journal of Biology, 70(1), 181-188.

Sazima, I. (1980). Behavior of two Brazilian species of parodontid fishes, Apareiodon piracicabae and $A$. ibitiensis. Copeia, 1, 166-169.

Selman, K., \& Wallace, R. A. (1989). Cellular aspects of oocyte growth in teleosts. Zoological Science, 6, 211-231.

Sokal, R. \& Rohlf, F. (1987). Introduction to Biostatistics. New York, USA: Freeman.

Suzuki, H. I., Vazzoler, A. E. A. de M., Marques, E. E., Lizama, M. de los A. P., Inada, P. (2004). Reproductive ecology of the fish assemblage. In S. M. Thomaz, A. A. Agostinho, \& N. S. Hahn (Eds.), The Upper Paraná River and its floodplain: physical aspects, ecology and conservation (pp. 271-292). Leiden, The Netherlands: Backhuys Publishers.

Thomé, R. G., Bazzoli, N., Rizzo, E., Santos, G. B. \& Ratton, F. T. (2005). Reproductive biology of Leporinus taeniatus Lütken (Pisces: Anostomidae) in Juramento Reservoir, Sao Francisco River Basin, Minas Gerais; Brazil. Revista Brasileira de Zoología, 22(3), 565-570.

Usma-Oviedo, J. S., Villa-Navarro, F., Lasso-Alcalá, C. A., Castro, F., Zuñiga, P. T., Cipamocha, C. A., (...), \& Suárez, J. T. (2013). Peces dulceacuícolas migratorios de Colombia. In L. A. Zapata-Padilla \& J. S. UsmaOviedo (Eds.), Guía de las Especies Migratorias de la Biodiversidad en Colombia (pp. 215-457). Bogotá,
Colombia: Ministerio de Ambiente y Desarrollo Sostenible / WWF-Colombia.

Usma-Oviedo, J. S., Ortega-Lara, A., \& Mancera-Rodríguez, N. J. (2012). Saccodon dariensis (Meek y Hildebrand 1913). In J. I. Mojica-Corzo, J. S. UsmaOviedo, R. Álvarez-León \& C. A. Lasso-Alcalá (Eds.), Libro Rojo de peces dulceacuícolas de Colombia (pp. 261-263). Bogotá, Colombia: Instituto de Investigación de Recursos Biológicos Alexander von Humboldt, Instituto de Ciencias Naturales de la Universidad Nacional de Colombia, WWF Colombia y Universidad de Manizales.

Vazzoler, A. E. A. de M. (1996). Biologia da reproducao de peixes teleosteos: teoria e pratica. Sao Pablo, Maringá: EDUEM.

Vazzoler, A. E. A. de M., \& Menezes, N. A. (1992). Síntese de conhecimento sobre o comportamento reprodutivo dos Characiformes da América do Sul (Teleostei, Ostariophysi). Revista Brasileira de Biología, 52(4), 627-640.

Welcomme, R. L. (1979). Fisheries ecology of floodplain rivers. London: Logman.

Wentworth, C. (1922). A scale of grade and class terms for clastic sediments. The Journal of Geology, 30, 377-392.

Wiley, M. L., \& Collette, B. B. (1970). Breeding tubercles and contact organs in fishes: their occurrence, structure and significance. Bulletin of the American Museum of Natural History, 143, 146-215.

Winemiller, K. O. (1989). Patterns of variation in life history among South American fishes in seasonal environments. Oecologia, 81, 225-241.

Wootton, R. J. (1984). Introduction: strategies and tactics in fish reproduction. In G. W. Potts \& R. J. Wootton (Eds.), Fish reproduction strategies and tactics (pp. 1-12). Orlando: Academic Press.

Zar, J. H. (1999). Biostatistical Analysis. New Jersey, USA: Prentice-Hall. 
